\title{
Biodegradation of Dimethyl Phthalate by Freshwater Unicellular Cyanobacteria
}

\author{
Xiaohui Zhang, ${ }^{1}$ Lincong Liu, ${ }^{1}$ Siping Zhang, ${ }^{1}$ Yan Pan, ${ }^{1}$ Jing Li, ${ }^{1}$ Hongwei Pan, \\ Shiguo $\mathrm{Xu}^{2}{ }^{2}$ and Feng Luo ${ }^{1}$ \\ ${ }^{1}$ Research Center of Bioenergy and Bioremediation, College of Resource and Environment, Southwest University, \\ Chongqing 400715, China \\ ${ }^{2}$ Institute of Environment and Ecology, Shandong Normal University, 88 Wenhua Donglu, Jinan, Shandong 250014, China \\ Correspondence should be addressed to Shiguo Xu; mailxsg@126.com and Feng Luo; van77@swu.edu.cn
}

Received 2 September 2016; Revised 5 November 2016; Accepted 21 November 2016

Academic Editor: Chunlei Xia

Copyright (c) 2016 Xiaohui Zhang et al. This is an open access article distributed under the Creative Commons Attribution License, which permits unrestricted use, distribution, and reproduction in any medium, provided the original work is properly cited.

\begin{abstract}
The biodegradation characteristics of dimethyl phthalate (DMP) by three freshwater unicellular organisms were investigated in this study. The findings revealed that all the organisms were capable of metabolizing DMP; among them, Cyanothece sp. PCC7822 achieved the highest degradation efficiency. Lower concentration of DMP supported the growth of the Cyanobacteria; however, with the increase of DMP concentration growth of Cyanobacteria was inhibited remarkably. Phthalic acid (PA) was detected to be an intermediate degradation product of DMP and accumulated in the culture solution. The optimal initial $\mathrm{pH}$ value for the degradation was detected to be 9.0, which mitigated the decrease of $\mathrm{pH}$ resulting from the production of PA. The optimum temperature for DMP degradation of the three species of organisms is $30^{\circ} \mathrm{C}$. After 72 hours' incubation, no more than $11.8 \%$ of the residual of DMP aggregated in Cyanobacteria cells while majority of DMP remained in the medium. Moreover, esterase was induced by DMP and the activity kept increasing during the degradation process. This suggested that esterase could assist in the degradation of DMP.
\end{abstract}

\section{Introduction}

Phthalic acid esters (PAEs) are a class of refractory organic plasticizer compounds that are widely utilized as additives and plasticizers in a variety of polymeric products, including paints, cosmetics, and flexible plastics, such as plastic toys and containers [1-3]. Consequently, they are found spreading ubiquitously at high concentrations in terrestrial and aquatic ecosystems [4-6]. In addition, PAEs are endocrine-disrupting pollutants and can show adverse effects on the environment and human health, inducing hepatic peroxisome proliferation, hormonal disorders, reproductive toxicity, and carcinogenicity $[7,8]$. They are classified as priority pollutant in the United States, the European Union, and China $[6,9,10]$. Dimethyl phthalate (DMP) is one kind of PAEs and the solubility in water is one of the highest among all the PAEs, reaching $4500 \mathrm{mg} \mathrm{L}^{-1}$ at $25^{\circ} \mathrm{C}$; inevitably, it could accumulate in the aquatic systems. Although DMP has only a moderate toxicity, its metabolic intermediate mono-methylphthalate (MMP) is not only toxic but also an endocrine disruptor and may interfere with the development and reproductive system of animals, or even human, by reducing testosterone production and decreased sperm counts [11]. Subsequently, MMP can be further degraded into phthalic acid (PA) and finally $\mathrm{CO}_{2}$ and $\mathrm{H}_{2} \mathrm{O}$ [12]. Due to the wide distribution, persistence, and difficulty in removal of PAEs, treatment approaches for PAEscontaminated wastewater have attracted many researchers' attention [13-15]. Different methods for removing PAEs from the environment include physical, chemical, and biological treatments, advanced oxidation processes, and combinations of these techniques [16-20]. Unfortunately, these methods are limited in application because of the high cost. In recent years, a number of studies have been carried out to evaluate the degradation of PAEs by bacteria in terrestrial and aquatic ecosystems $[12,21]$. Cyanobacteria are a class of autotrophic microorganisms capable of photosynthesis. Cyanobacteria have a high photosynthetic efficiency (upwards of $10 \%$ ), 
significantly better than land plants $(\sim 3-4 \%$ maximum efficiency) $[22,23]$. As the primary producers, Cyanobacteria and microalgae support more than half of the global primary production in the aquatic food web. Pollutants can enter aquatic food chains through bioaccumulation of Cyanobacteria and microalgae, so their response will certainly affect upper tropic levels. Moreover, it has been well known that microorganism degradation is a vital process affecting the environmental fate of PAEs [20, 24] In addition to producing oxygen to meet the needs of heterotrophic bacteria and subsequently stimulate the activities of the bacteria degrading organic pollutants, Cyanobacteria and microalgae are also capable of degrading organic pollutants directly, such as phenolics, polycyclic aromatic hydrocarbons, pesticides, petroleum, and PAEs [25]. However, most of those studies have focused on determining either optimizing conditions of PAEs or the degradation efficiency in some groups of organisms. Comprehensive degradation characters of aquatic prokaryotes have not yet been elucidated. Moreover, genome sequences and genetic manipulation techniques are insufficient for the Cyanobacteria used in the previous studies.

Therefore, characteristics of DMP biodegradation of three model freshwater unicellular Cyanobacteria, including two nonnitrogen fixing Cyanobacteria Syenchocystis sp. PCC6803, Synechococcus sp. PCC7942 and a diazotrophic one, Cyanothece sp. PCC7822, were illuminated. Most importantly, the three photoautotrophic Cyanobacteria have the advantages that their genome sequences are available and all the three species can be manipulated by genetic engineering [26-28]. The objective of this study is to investigate the effect of DMP on the growth of Cyanobacteria and to characterize the DMP biodegradation of the three Cyanobacteria. Furthermore, the key enzyme activity was assayed. This study provides a novel idea to apply these three model Cyanobacteria to the bioremediation of PAEs and supplies basic knowledge for studying the mechanism in the PAEs degradation in molecular level in the near future.

\section{Materials and Methods}

2.1. Chemicals and Cyanobacteria. Dimethyl phthalate (DMP), mono-methylphthalate (MMP), phthalic acid (PA), and 4-nitrophenyl acetate were purchased from SigmaAldrich (Steinem, Germany). Bio-Rad Protein Assay Kit II was purchased from Bio-Rad Company (Hercules, Calif. USA). Three species of freshwater unicellular Cyanobacteria were provided by Purdue University, West Lafayette, USA, including two nonnitrogen fixing Cyanobacteria Synechocystis sp. PCC6803, Synechococcus sp. PCC7942, and a diazotrophic Cyanothece sp. PCC7822. $100 \mathrm{~mL}$ of Cyanobacteria cells was cultivated in sterilized BG11 medium in $250 \mathrm{~mL}$ flasks in the laboratory at $25 \pm 1^{\circ} \mathrm{C}$ under a $16: 8$ light: dark cycle provided by cool white fluorescent tubes at a light intensity of $50 \mu \mathrm{mol}$ photons $\mathrm{m}^{-2} \mathrm{~s}^{-1}$. Prior to being used for the experiments, the cells were maintained in the exponential growth phase by repeated inoculation into fresh medium. All samples were prepared in triplicate and incubated using a rotary shaker operated at $100 \mathrm{rpm}$.
2.2. Effect of DMP on the Growth of Cyanobacteria. To evaluate the effect of DMP on the growth of Cyanobacteria, the growth curves of three Cyanobacteria under a variety of concentrations of DMP were investigated. Cyanobacteria cells in the middle exponential phase of growth were harvested by centrifugation ( $10000 \mathrm{~g}, 10 \mathrm{~min})$. The collected cells were washed twice with BG11 medium for experiments. A stock solution of DMP $4000 \mathrm{mg} \mathrm{L}^{-1}$ in BG11 medium was prepared. Different concentrations of DMP 0, 20, 50, 100, 200, and $500 \mathrm{mg} \mathrm{L}^{-1}$ in the culture were obtained by diluting the stock solution with BG11 medium. Growth was determined every $24 \mathrm{~h}$ for up to 120 hours, based on the absorbance at $730 \mathrm{~nm}\left(\mathrm{OD}_{730}\right)$ using a UV-vis spectrophotometer Shimadzu UV2600 (Shimadzu, Kyoto, Japan). The initial $\mathrm{OD}_{730}$ of the Cyanobacteria culture were set around 0.10 and cells were cultivated at the same condition as the preculture. $2 \mathrm{~mL}$ cells were taken every $24 \mathrm{~h}$ for sampling at time points of 0 , $24,48,72,96$, and $120 \mathrm{~h}$.

2.3. Biodegradation of DMP by Cyanobacteria. The stock solution of DMP was diluted with sterilized BG11 medium to reach DMP of 20 and $50 \mathrm{mg} \mathrm{L}^{-1}$. The final cell density of each Cyanobacteria species was measuring the optical density at $\mathrm{OD}_{730}$ around 0.15 . Supernatant separated from triplicate flasks of each culture every 24 hours was utilized for the DMP measurement. The DMP extraction treatment included centrifuging the culture for $10,000 \mathrm{~g}$ for $10 \mathrm{~min}$ and filtrating the supernatant through a $0.45 \mu \mathrm{m}$ pore-size filter for HPLC assay.

2.4. Effect of Temperature on the DMP Biodegradation. A series of temperatures $15^{\circ} \mathrm{C}, 25^{\circ} \mathrm{C}, 30^{\circ} \mathrm{C}, 35^{\circ} \mathrm{C}$, and $40^{\circ} \mathrm{C}$ were set to compare the degradation efficiency of the three species of Cyanobacteria. The initial $\mathrm{OD}_{730}$ of the Cyanobacteria culture were set around 0.10 and cells were cultivated at the same condition as the preculture. Cultures with $50 \mathrm{mg} \mathrm{L}^{-1}$ of DMP were inoculated in growth chambers (RDN-400C-4, Dongnan Co. Ltd., China) provided with LED light intensity of $50 \mu \mathrm{mol}$ photons $\mathrm{m}^{-2} \mathrm{~s}^{-1}$ under a $16: 8$ light : dark cycle. After $96 \mathrm{~h}$, the DMP degradation efficiency was calculated.

2.5. Effect of Initial $\mathrm{pH}$ on the Biodegradation. The $\mathrm{pH}$ values of the culture medium of Cyanothece sp. PCC7822 with 20 and $50 \mathrm{mg} \mathrm{L}^{-1}$ DMP were measured every $24 \mathrm{~h}$ for 120 hours. $10 \mathrm{~mL}$ culture samples were taken for $\mathrm{pH}$ measurement. Simultaneously, the PA accumulation in same cultures was investigated and PA concentration was assayed by using HPLC methods. A series of initial $\mathrm{pH}$ of 6.0, 7.0, 8.0, 9.0, and 10.0 in the BG11 medium amended with $50 \mathrm{mg} \mathrm{L}^{-1} \mathrm{DMP}$ were adjusted by $\mathrm{HCl}$ and $\mathrm{NaOH}$. Cells with an intensity of $\mathrm{OD}_{730} 0.15$ were inoculated in the medium for the study, DMP degradation efficiency was calculated under different initial $\mathrm{pH}$ conditions after $72 \mathrm{~h}$.

2.6. High Performance Liquid Chromatography (HPLC) Anal$y$ sis. For the analysis of extracellular residual DMP, MMP, and $\mathrm{PA}$ in the solution, $2 \mathrm{~mL}$ of Cyanobacteria solution was centrifuged $(10,000 \mathrm{rpm}, 10 \mathrm{~min})$. The supernatant was 
collected and filtered through a $0.45 \mu \mathrm{m}$ pore-size filter for analysis in HPLC. For the DMP concentrations in vivo, cells were harvested from $20 \mathrm{~mL}$ of culture medium by centrifugation at $10,000 \mathrm{~g}$ for $10 \mathrm{~min}$. Then the pellet was disrupted by grinding with liquid nitrogen and suspended with $2 \mathrm{~mL}$ dichloromethane at $5000 \mathrm{rpm}$ for $10 \mathrm{~min}$. The solution was centrifuged at $4000 \mathrm{rpm}$ for $10 \mathrm{~min}$. After discarding of the water phase, the dichloromethane layer was concentrated to a final volume of $0.5 \mathrm{~mL}$. An analytical high performance liquid chromatography LC-20AD (Shimadzu, Kyoto, Japan) was used to analyze the DMP and its metabolites DMP, MMP, and PA. Samples were analyzed by a capillary column InertSustain C18 $(4.6 \mathrm{~mm} \times 150 \mathrm{~mm} \times 5 \mu \mathrm{m})$; The mobile phase used was methanol- $0.1 \%$ phosphoric acid solution $(50: 50, \mathrm{v} / \mathrm{v})$ with a flow rate of $1.0 \mathrm{~mL} / \mathrm{min}$. The sample volume of $20 \mu \mathrm{L}$ was injected into an oven temperature $30^{\circ} \mathrm{C}$, with a UV detector, and detecting wavelength was set at $226 \mathrm{~nm}$. The retention times of DMP MMP and PA were identified according to the retention time of the reference standards.

2.7. Esterase Activity Assay. Cyanobacterial cells were cultivated with $50 \mathrm{mg} \mathrm{L}^{-1}$ DMP or without DMP as a control. Cells were harvested by centrifugation at $10,000 \mathrm{~g}$ for $10 \mathrm{~min}$ at $4^{\circ} \mathrm{C}$, after three times washing with $50 \mathrm{mM}$ potassium phosphate ( $\mathrm{pH} 7.0$ ), the pellet was disrupted by grinding with liquid nitrogen and suspended in the same buffer followed by centrifuge for $10 \mathrm{mins}$ at $4^{\circ} \mathrm{C}$, and supernatant was then subject to the enzyme assay, as described previously [29, $30]$ with some modifications. The reaction mixture included $50 \mathrm{mM}$ of potassium phosphate buffer at $\mathrm{pH} 7.0$ with an appropriate amount of cell-free enzyme extract and $100 \mu \mathrm{L}$ of $25 \mathrm{mM}$ 4-nitrophenyl acetate diluted to a total volume of $1 \mathrm{~mL}$ at $25^{\circ} \mathrm{C}$. The protein content was estimated using the Bio-Rad Protein Assay Kit II using BSA as the standard according to the manufacturer's instruction. The reaction was stopped by adding $50 \mu \mathrm{L}$ of $2 \mathrm{~N} \mathrm{HCL}$. The optical density at $400 \mathrm{~nm}$ of reaction mixture was measured using a UV-vis spectrophotometer UV2600 (Shimadzu, Kyoto, Japan). A blank was measured using only buffer. The increase of the absorbance at $400 \mathrm{~nm}$ implies the increase of 4-nitrophenolate as a product of the reaction resulted from the hydrolysis of 4-nitrophenyl acetate. The enzyme activity was presented in the unit of $\mathrm{U} \mathrm{mL}^{-1}$ as in the previous studies [30]. Experimental data was analyzed with SPSS software (version 17.0).

\section{Results and Discussion}

3.1. Correlations between DMP Concentration and Cyanobacteria Growth. Phthalic acid esters are endocrine-disrupting compounds, which have been shown to reduce the diversity of microbial communities and decline crop quality [31], and are toxic to most aquatic organisms including algae [32]. Higher concentration of DMP inhibits the growth rate, while lower concentration can induce the growth of Cyanobacteria or algae $[33,34]$. However, it was also reported that, even at as high as $200 \mathrm{mg} \mathrm{L}^{-1}$, PAEs shows a promotive effect on the growth of Cyanobacteria [30]. In this study, DMP was introduced to BG11 medium at concentrations of 0 ,
$20,50,200$, and $500 \mathrm{mg} \mathrm{L}^{-1}$, and its effects on the growth of three Cyanobacteria were evaluated over 120 hours. A growth-stimulative effect of DMP was found only at the concentration of $20 \mathrm{mg} \mathrm{L}^{-1}$, but higher concentration of DMP ( $\geq 50 \mathrm{mg} \mathrm{L}^{-1}$ ) revealed growth inhibition activity with increasing concentrations of DMP during the study period $120 \mathrm{~h}$. Figure 1 shows the typical growth patterns of all three Cyanobacteria in the absence or presence of each concentration of DMP. However, with the increase of the concentration of DMP, three Cyanobacteria showed different degree of growth inhibition and all showed serious growth deduction at higher concentration. The growth of Synechocystis was inhibited completely at the DMP concentration of $500 \mathrm{mg} \mathrm{L}^{-1}$ (Figure 1(a)). Synechococcus was more sensitive to higher concentration of DMP; $200 \mathrm{mg} \mathrm{L}^{-1}$ of DMP suppressed the growth entirely (Figure 1(b)). Among them, Cyanothece sp. 7822 showed the most tolerance to higher concentration of DMP; its growth maintained to continue under the highest concentration applied (Figure 1(c)). Different Cyanobacteria showed different tolerance to the DMP; the reason could be due to their surface composition and surface area exposed to the DMP. For instance, Cyanothece sp. PCC7822 has a thicker layer of extracellular polysaccharide, which may aid the cells to tolerate the toxicity in the environments. The intracellular and extracellular enzymes involved in the degradation of DMP are also important in supporting the cells that survive in the stress conditions.

Additionally, the maximum growth rate of the three Cyanobacteria was observed in the presence of $20 \mathrm{mg} \mathrm{L}^{-1}$ DMP; this suggests that low concentration DMP are taken up and degraded by the cyanobacterial cells and finally used as a carbon source. These results revealed a significant difference between the samples incubated in different concentrations of DMP. A positive correlation was observed between the $20 \mathrm{mg} \mathrm{L}^{-1}$ DMP and growth rate in all three species and a negative correlation between growth and high DMP concentration.

3.2. Biodegradation of DMP. The biodegradation tendency of DMP was studied by incubating cyanobacterial cultures with 20 and $50 \mathrm{mg} \mathrm{L}^{-1}$ of DMP and then measuring the DMP that remained in the culture medium every 24 hours during the incubation process, as shown in Figure 2. There was a similar decreasing trend of DMP concentration over time in all three species. However, DMP degraded more rapidly in cultures incubated with Cyanothece than in the other two cultures. Apparently, there was no trace amount of DMP detected in Cyanothece culture after incubation for 96 hours with an initial concentration of $20 \mathrm{mg} \mathrm{L}^{-1}$ and 120 hours with $50 \mathrm{mg} \mathrm{L}^{-1}$ of DMP. The time course of biodegradation of DMP was similar in the three Cyanobacteria and the degradation tendency of the three Cyanobacteria is equivalent to that of the microalgae $D$. tertiolecta [33]. The degradation rate was slow at the beginning and gradually increased with the incubation time; eventually it reached highest at the time span from $72 \mathrm{~h}$ to $96 \mathrm{~h}$. In the cultures with initial concentration of $20 \mathrm{mg} \mathrm{L}^{-1}$ the degradation rate was $0.357 \mathrm{mg} \mathrm{L}^{-1} \mathrm{~h}^{-1}$ for Cyanothece and $0.271 \mathrm{mg} \mathrm{L}^{-1} \mathrm{~h}^{-1}$ for Synechococcus. In the cultures with 


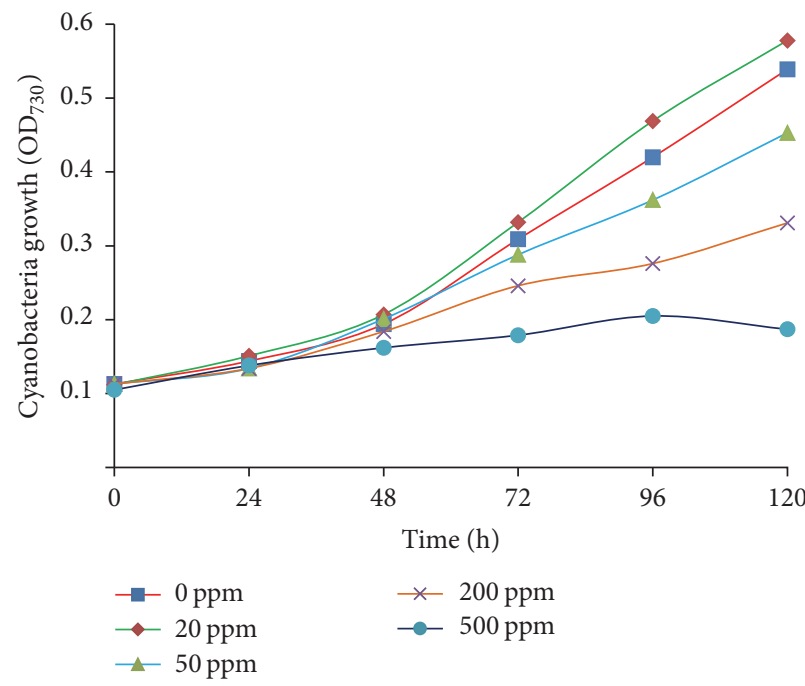

(a)

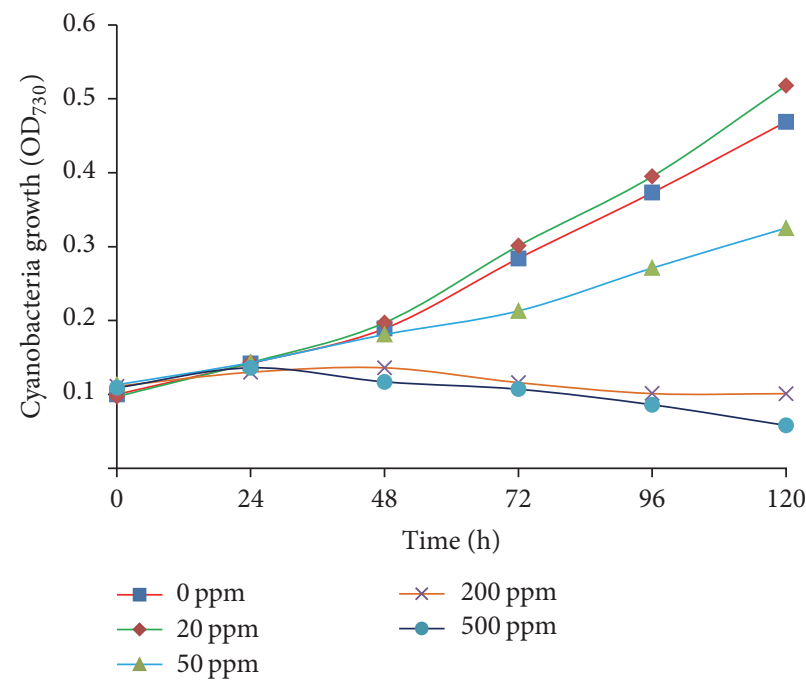

(b)

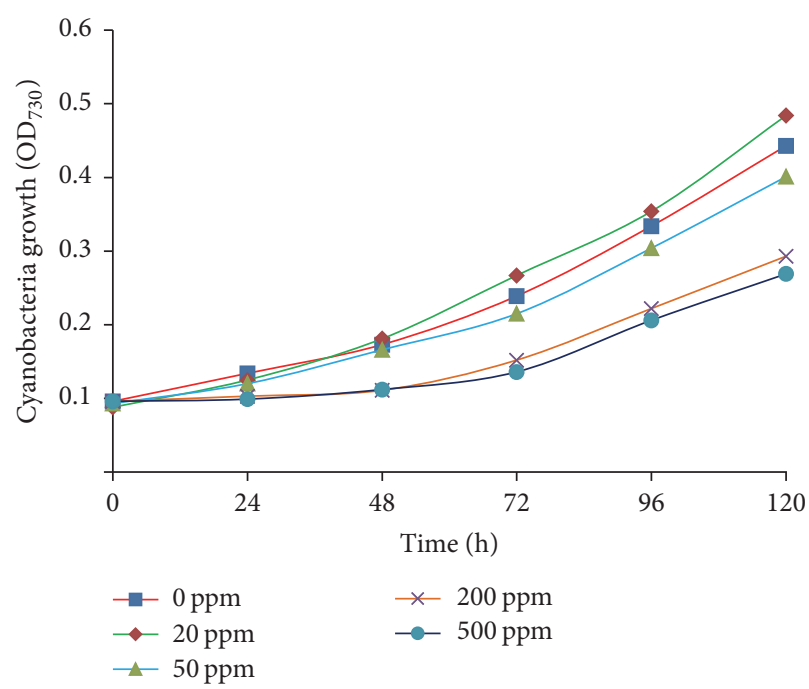

(c)

FIGURE 1: Effect of different concentration of DMP on the growth of three Cyanobacteria. (a) Synechocystis sp. PCC6803 (6803), (b) Synechococcus sp. PCC7942 (7942), and (c) Cyanothece sp. PCC7822 (7822).

initial concentration of $50 \mathrm{mg} \mathrm{L}^{-1}$, although the Cyanothece completed the degradation earlier, Synechocystis showed the highest degradation rate $0.6746 \mathrm{mg} \mathrm{L}^{-1} \mathrm{~h}^{-1}$, slightly higher than Cyanothece $0.5567 \mathrm{mg} \mathrm{L}^{-1} \mathrm{~h}^{-1}$.

During the degradation of DMP, Cyanothece displayed the ability to tolerate and degrade the DMP more efficiently than the other two species of Cyanobacteria. Synechocystis showed a moderate biodegradation ability and Synechococcus showed the lowest. The highest degradation rate of Cyanothece is $8.75 \mathrm{mg} \mathrm{L}^{-1} \mathrm{~d}^{-1}$ in the initial DMP concentration $20 \mathrm{mg} \mathrm{L}^{-1}$ and $15.85 \mathrm{mg} \mathrm{L}^{-1} \mathrm{~d}^{-1}$ in the initial concentration $50 \mathrm{mg} \mathrm{L}^{-1}$, which is comparable to that of Dunaliella exhibited biodegradation rates of $11.3-30.5 \mathrm{mg} \mathrm{L}^{-1} \mathrm{~d}^{-1}$ for $100-$ $300 \mathrm{mg} \mathrm{L}^{-1}$ of DMP [33]. Chlorella pyrenoidosa showed a lower biodegradation rate with average biodegradation rates between 2.1 and $13.4 \mathrm{mg} \mathrm{L}^{-1} \mathrm{~d}^{-1}$ being observed for DMP [35].
During the biodegradation of DMP, organisms such as the green alga Chlorella vulgaris [36] and bacteria [37, 38] generally follow a first-order reaction model, while some other green algae such as Dunaliella tertiolecta [33] follow a second-order reaction pattern. The results of our present study showed the pattern of DMP degradation of Cyanobacteria is more likely to be a second-order reaction model (Figure 2). The degradation products were subsequently analyzed with HPLC. DMP and its intermediate degradation metabolites MMP and PA were shown in Figure S1 (see Supplementary Material available online at http://dx.doi.org/10.1155/2016/5178697). The DMP was detected at a retention time of $8.278 \mathrm{~min}$ and the compound detected at a retention time of $4.567 \mathrm{~min}$ was identical to that of authentic MMP and 3.098 min was identical to that of PA in the degradation products. 


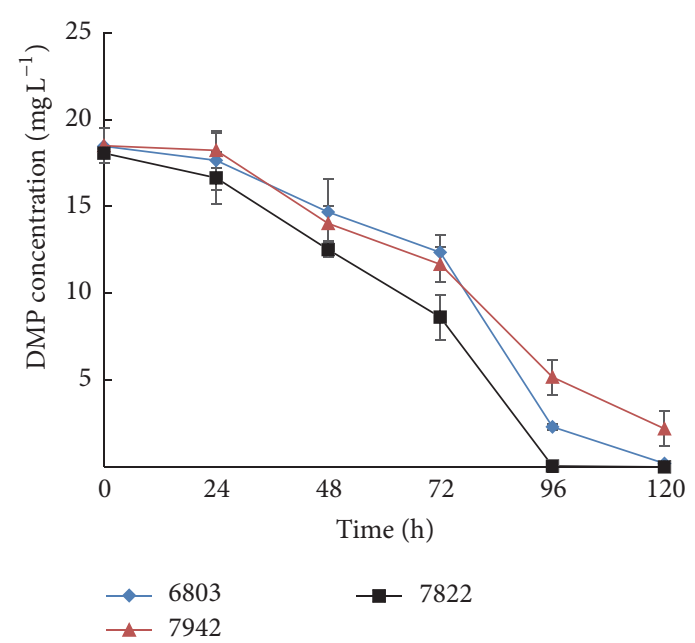

(a)

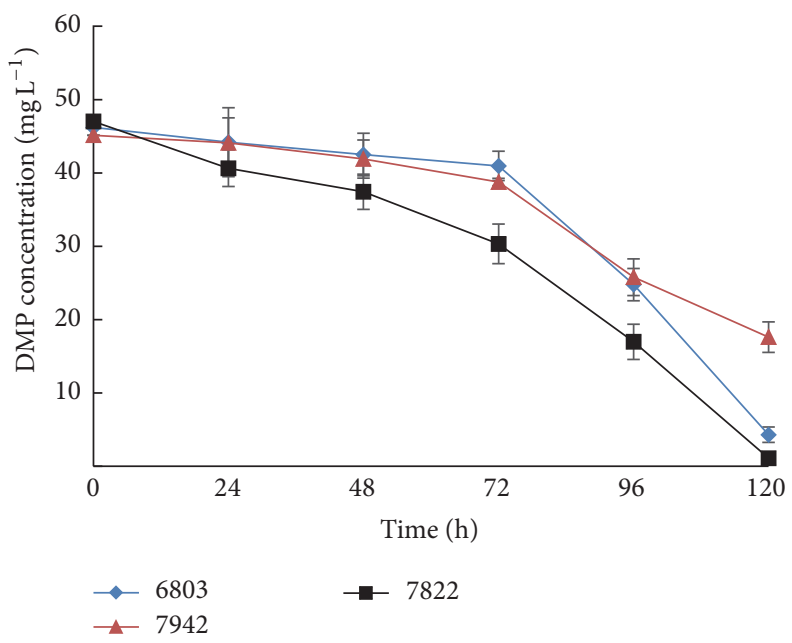

(b)

Figure 2: Change of the DMP concentrations of the culture medium over time by Synechocystis sp. PCC6803 (6803), Synechococcus sp. PCC7942 (7942), and Cyanothece sp. PCC7822 (7822). With an initial DMP concentration around $20 \mathrm{mg} \mathrm{L}^{-1}$ (a) and $50 \mathrm{mg} \mathrm{L}^{-1}$ (b).

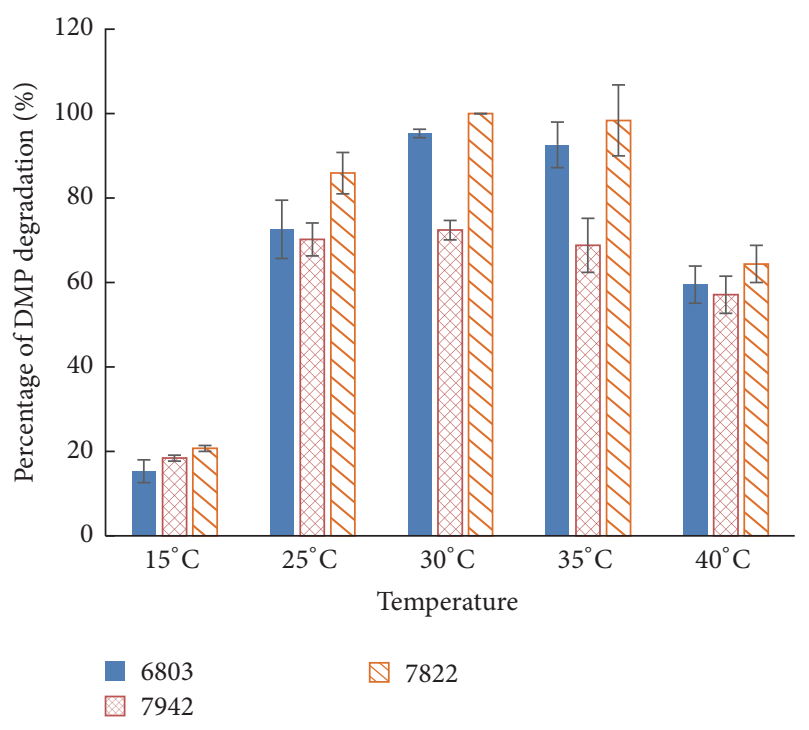

FIGURE 3: The influence of temperature on the efficiency of DMP biodegradation by Synechocystis sp. PCC6803 (6803), Synechococcus sp. PCC7942 (7942), and Cyanothece sp. PCC7822 (7822).

\subsection{Effect of Temperature on the DMP Biodegradation. Sim-} ilar as the other microorganisms, the DMP degradation of Cyanobacteria is influenced by the factor of temperature. The data in Figure 3 showed the dependence of the degradation efficiency on the temperature. After $96 \mathrm{~h}$ incubation, almost all the DMP of $50 \mathrm{mg} \mathrm{L}^{-1}$ in the cultures was degraded at $30^{\circ} \mathrm{C}$ and $35^{\circ} \mathrm{C}$. The degradation efficiency of the three species of Cyanobacteria at $30^{\circ} \mathrm{C}$ is slightly higher than that at $35^{\circ} \mathrm{C}$ and Cyanothece sp. PCC7822 finished the degradation of DMP completely. At $15^{\circ} \mathrm{C}$, three Cyanobacteria performed the lowest degradation ability, less than $20 \%$. Surprisingly, at $40^{\circ} \mathrm{C}$, Cyanobacteria still remained to have active degradation abilities; even though the cells appeared yellowish, more than
$60 \%$ of the DMP was degraded, comparable to $25^{\circ} \mathrm{C}$. In the previous studies, no matter bacteria or microalgae, experimental temperatures were set at around $25^{\circ} \mathrm{C}[11,30,35-37]$, taking the environmental temperature into consideration. However, at $25^{\circ} \mathrm{C}$, the degradation of DMP of the three Cyanobacteria is about $50-60 \%$ of that at $30^{\circ} \mathrm{C}$. Optimum temperature for the three species of Cyanobacteria to degrade $\mathrm{DMP}$ is $30^{\circ} \mathrm{C}$ and at this temperature, cells condition appears healthy.

3.4. Accumulation of $P A$ and Change of $p H$. During the process of incubation, DMP was degraded by Cyanothece and the PA as an intermediate product accumulated in culture medium. The PA concentrations gradually increased, which is in direct proportion to initial DMP concentration. The highest net production of PA was also between hours 72 and 96 during the degradation (Figure 4(a)). Within the most efficient period, net PA production speed was 0.039 and $0.069 \mathrm{mg} \mathrm{L}^{-1} \mathrm{~h}^{-1}$ with DMP initial concentration of 20 and $50 \mathrm{mg} \mathrm{L}^{-1}$. Distribution patterns of DMP in three Cyanobacteria culture at 72 hour are shown in Figure 5. The residual DMP mainly presents in medium (extracellular) and no more than $11.8 \%$ was in Cyanothece cell (intracellular) phase. In Synechocystis the extracellular DMP occupied only $8.5 \%$ and for Synechococcus only 6.3\%.

The accumulation of PA resulted in the deduction of the $\mathrm{pH}$. As shown in Figure 4(a) the $\mathrm{pH}$ values of the culture with $20 \mathrm{mg} \mathrm{L}^{-1}$ DMP are always higher than that with $50 \mathrm{mg} \mathrm{L}^{-1}$ DMP. The initial pH of BG11 medium is around 7.2. After the 120 hours' incubation, $\mathrm{pH}$ values increased 0.94 and 0.81 , respectively. To investigate the optimum initial $\mathrm{pH}$ for the DMP biodegradation, a variety of $\mathrm{pH}$ values were set ranging from 6.0 to 10.0 and the Cyanobacteria showed different efficiency in the DMP degradation. In the culture with initial $\mathrm{pH}$ 6.0, three species of Cyanobacteria showed the lowest degradation functions. Initial $\mathrm{pH} 9.0$ is the optimum value for 


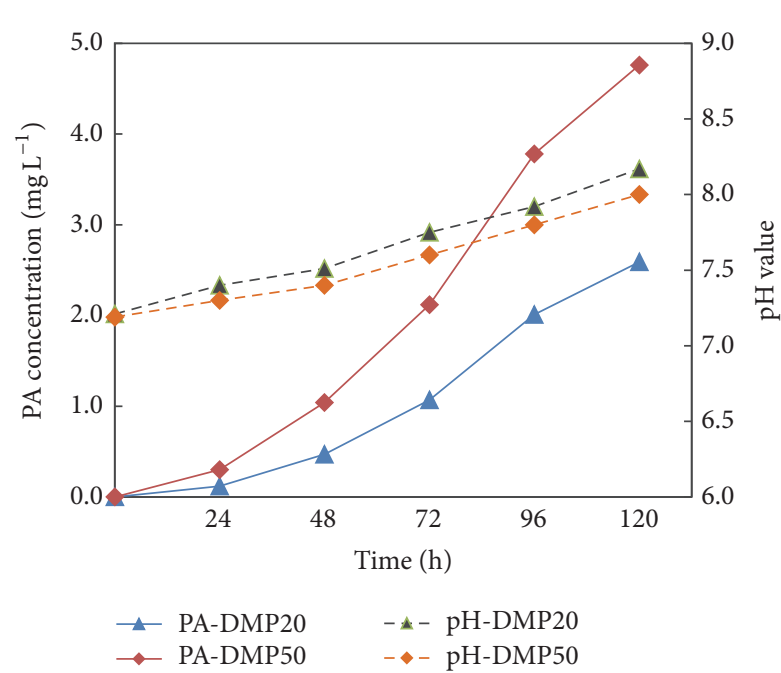

(a)

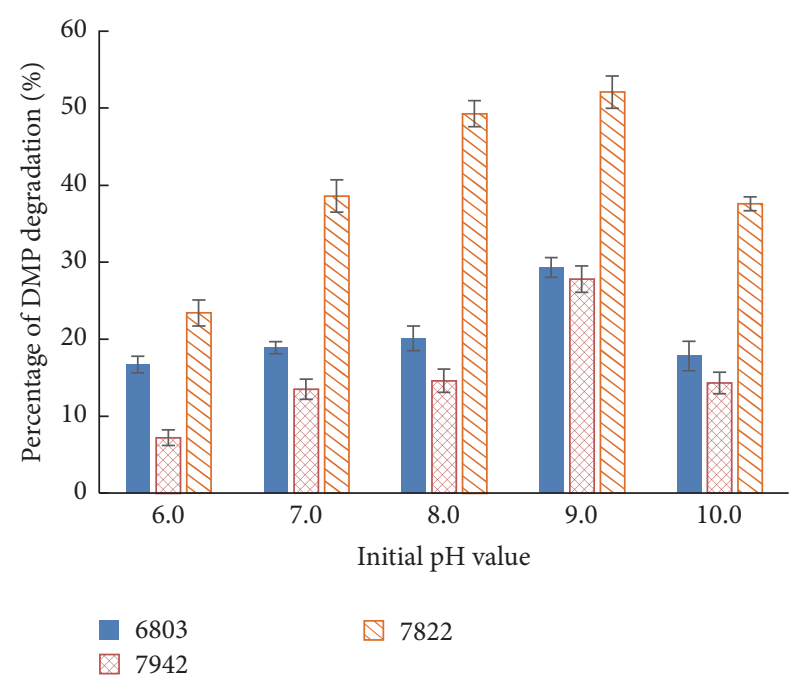

(b)

FIGURE 4: The process of PA accumulation and pH change of the Cyanothece sp. PCC7822 (a) and the influence of initial pH on the efficiency of DMP biodegradation by Synechocystis sp. PCC6803 (6803), Synechococcus sp. PCC7942 (7942), and Cyanothece sp. PCC7822 (7822) (b).

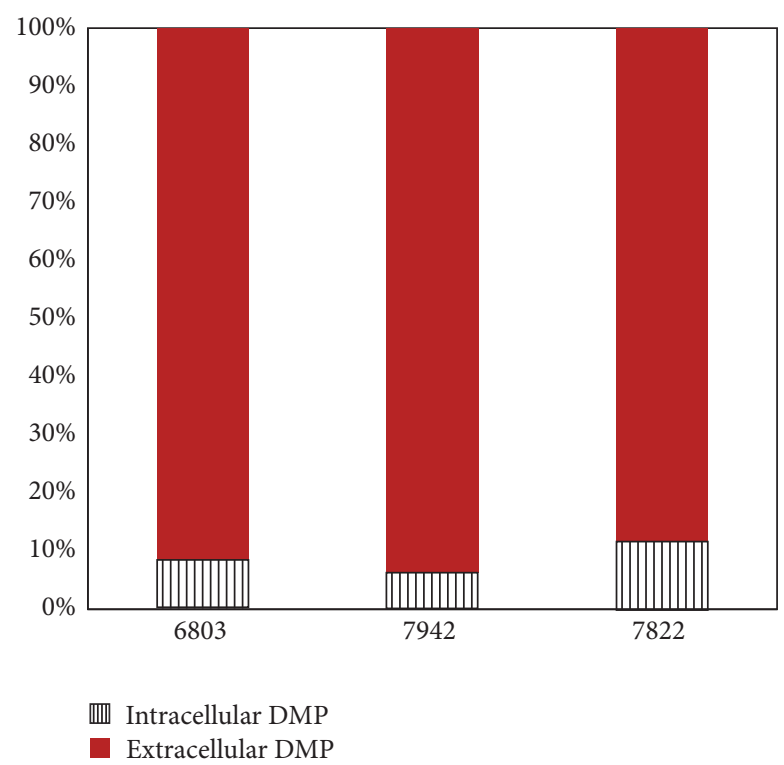

FIGURE 5: Percentages of residual DMP distributed in Synechocystis sp. PCC6803 (6803), Synechococcus sp. PCC7942 (7942), and Cyanothece sp. PCC7822 (7822) cells (intracellular DMP), and in the medium (extracellular DMP) incubated with $50 \mathrm{mg} \mathrm{L}^{-1}$ DMP for 72 hours.

DMP degradation (Figure 4(b)). Generally speaking, during growth of Cyanobacteria $\mathrm{pH}$ values of the cultures increase gradually due to the assimilation of $\mathrm{NaHCO}_{3}$ and $\mathrm{NaNO}_{3}$ and as carbon and nitrogen source, and alkaline medium is more suitable for the Cyanobacteria growth; however, with the accumulation of $\mathrm{PA}$, the $\mathrm{pH}$ increase was deducted. The inhibition of Cyanobacteria growth showed in Figure 1 is not only due to the toxicity of DMP but also caused by its intermediate metabolites MMP and PA (Figure 4).
3.5. Enzyme Activity for DMP Degradation. The biodegradation efficiency for DMP was evaluated in vitro using enzyme extracts from Cyanobacteria from the culture amended with or without DMP. As shown in Table 1, the esterase activities were significantly lower in all three Cyanobacteria grown in the absence of DMP. However, an obvious elevation was observed when cells were grown in the presence of DMP. As shown in Table 1, after 72 hours' incubation, the esterase activities of Cyanobacteria without DMP were equivalent to that of the cells just being introduced with DMP. However, a drastic elevation in enzyme activity was observed with the increase of incubation time when cells were in the presence of DMP. From $0 \mathrm{~h}$ to $72 \mathrm{~h}$ after being exposed to DMP, the esterase activity increased $14.5-160.0$ times. The activities at 24 hour were only $28.1-31.5 \%$ of the activity at 72 hours. Similar phenomena were observed in all three tested organisms and a slightly higher enzyme activity was observed for Cyanothece sp. PCC 7822.

The ester hydrolyzing enzymes (esterases) are a specified group of enzymes (EC 3.1.1.2), which are very vital for the primary attack of xenobiotics, including phthalic derivatives. Microbial degradation of PAEs is initiated by stepwise deesterification to form phthalate monoester and then PA sequentially [39]. Table 1 presents the catalysis profile of esterase activity of three Cyanobacteria during degradation of DMP. It was found that the presence of DMP resulted in the induction of esterases. Surprisingly, the esterase showed low activity in the culture right from the beginning at $50 \mathrm{mg} \mathrm{L}^{-1}$ DMP concentration; more interestingly, the activity was detected at 72 hours in the control as well. The esterase activity showed an increasing trend with increasing of incubation time and production of esterase by Cyanobacteria aided in the biodegradation of DMP. It was reported that deesterification is the first step in process of the DMP degradation of the Bacillus species, and two of the four isoesterases had very good DMP-hydrolyzing ability [40]. The esterase activity 
TABLE 1: Esterase activity profiles for cyanobacteria Syenchocystis sp. PCC6803 (6803), Synechococcous sp. PCC7942 (7942), and Cyanothece sp. PCC7822 (7822) at different incubation time, with $50 \mathrm{mg} \mathrm{L}^{-1} \mathrm{DMP}(+\mathrm{DMP})$ or without DMP (-DMP).

\begin{tabular}{lcccr}
\hline Organism & +DMP & \multicolumn{2}{c}{$-\mathrm{DMP}$} & \\
& $0 \mathrm{~h}$ & $24 \mathrm{~h}$ & $72 \mathrm{~h}$ & $0.09 \pm 0.01$ \\
6803 & $0.06 \pm 0.01$ & $0.27 \pm 0.03$ & $0.96 \pm 0.11$ & $0.13 \pm 0.02$ \\
7942 & $0.06 \pm 0.01$ & $0.26 \pm 0.04$ & $0.87 \pm 0.06$ & $0.10 \pm 0.02$ \\
7822 & $0.07 \pm 0.02$ & $0.35 \pm 0.03$ & $1.11 \pm 0.09$ & \\
\hline
\end{tabular}

induced by DMP kept on increasing during the early period of degradation. This result also demonstrates that deesterification could be the initial step in the DMP degradation in Cyanobacteria.

Synechocystis sp. PCC 6803 is the first sequenced cyanobacterium [41]; subsequently, more cyanobacterial genome sequence was completed. The genome sequences of the three species of Cyanobacteria in this research are available (https://www.ncbi.nlm.nih.gov/). Five putative esterase genes were found in Synechocystis, sll0644, slr1916, sll0992, sll1284, and slr8023. Except for the defined esterase, four genes encoding probable esterases with the lotus tags synpcc7942_0451, synpcc7942_0458, synpcc7942_0774, and synpcc7942_2527 were detected in Synechococcus sp. PCC7942 as well as 3 genes with lotus tags Cyan7822_1061, Cyan7822_2840, and Cyan7822_3463 in Cyanothece sp. PCC7822. However, the expression and the functional esterase are remaining for further study.

\section{Conclusions}

Low concentration $20 \mathrm{mg} \mathrm{L}^{-1}$ DMP stimulated the growth of the three freshwater unicellular Cyanobacteria, but higher concentration could inhibited the growth. The Cyanobacteria investigated were capable of degrading DMP efficiently and Cyanothece was found to be the most effective species. PA as an intermediate degradation product of DMP accumulated in the Cyanobacteria culture and consequently caused the deduction of $\mathrm{pH} .30^{\circ} \mathrm{C}$ is optimum for the DMP degradation of Cyanobacteria. It was observed that only a small portion of DMP aggregated into the cells; majority still remained in the medium. Esterase was induced by DMP and the activity kept increasing during the degradation. Taken together, the results of the study demonstrate that the three model Cyanobacteria can be applied into DMP degradation in aquatic system. More importantly, this study also provides basic knowledge to study the degradation mechanism in the molecular level further.

\section{Competing Interests}

The authors declare that there is no conflict of interests regarding the publication of this paper.

\section{Authors' Contributions}

Xiaohui Zhang, Lincong Liu, and Siping Zhang contributed equally to this work.

\section{Acknowledgments}

This work is funded by Fundamental Research Funds for the Central Universities (XDJK2015C172, XDJK2016E164), National Natural Science Foundation of China (Grant no. 31500038), Doctoral Start-Up Grant of Southwest University (SWU115018), Chongqing Research Program of Basic Research and Frontier Technology (no. cstc2016jcyjA0540), and Projects of Scientific Platform and Base Construction (cstc2014pt-sy0017).

\section{References}

[1] J. Wang, Y. Luo, Y. Teng, W. Ma, P. Christie, and Z. Li, "Soil contamination by phthalate esters in Chinese intensive vegetable production systems with different modes of use of plastic film," Environmental Pollution, vol. 180, pp. 265-273, 2013.

[2] Z.-H. Luo, Y.-R. Wu, R. K. K. Chow, J.-J. Luo, J.-D. Gu, and L. L. P. Vrijmoed, "Purification and characterization of an intracellular esterase from a Fusarium species capable of degrading dimethyl terephthalate," Process Biochemistry, vol. 47, no. 5, pp. 687-693, 2012.

[3] W.-L. Wang, Q.-Y. Wu, C. Wang, T. He, and H.-Y. Hu, "Health risk assessment of phthalate esters (PAEs) in drinking water sources of China," Environmental Science and Pollution Research, vol. 22, no. 5, pp. 3620-3630, 2015.

[4] X.-Z. Meng, Y. Wang, N. Xiang et al., "Flow of sewage sludgeborne phthalate esters (PAEs) from human release to human intake: implication for risk assessment of sludge applied to soil," Science of the Total Environment, vol. 476-477, pp. 242-249, 2014.

[5] E. Psillakis, D. Mantzavinos, and N. Kalogerakis, "Monitoring the sonochemical degradation of phthalate esters in water using solid-phase microextraction," Chemosphere, vol. 54, no. 7, pp. 849-857, 2004.

[6] T. Xiaoyan, W. Suyu, Y. Yang et al., "Removal of six phthalic acid esters (PAEs) from domestic sewage by constructed wetlands," Chemical Engineering Journal, vol. 275, pp. 198-205, 2015.

[7] P. Hsu, Y. Kuo, Y. Leon Guo et al., "The adverse effects of low-dose exposure to $\mathrm{Di}$ (2-ethylhexyl) phthalate during adolescence on sperm function in adult rats," Environmental Toxicology, vol. 31, no. 6, pp. 706-712, 2016.

[8] I. Rusyn, J. Peters, and M. Cunningham, "Modes of action and species-specific effects of di-(2-ethylhexyl)phthalate in the liver," Critical Reviews in Toxicology, vol. 36, no. 5, pp. 459-479, 2006.

[9] W. Jianlong, L. Ping, and Q. Yi, "Microbial degradation of din-butyl phthalate," Chemosphere, vol. 31, no. 9, pp. 4051-4056, 1995.

[10] L. Zhang, J. Liu, H. Liu, G. Wan, and S. Zhang, “The occurrence and ecological risk assessment of phthalate esters (PAEs) in 
urban aquatic environments of China," Ecotoxicology, vol. 24, no. 5, pp. 967-984, 2015.

[11] S. K. Brar, M. Verma, R. D. Tyagi, J. R. Valéro, and R. Y. Surampalli, "Concurrent degradation of dimethyl phthalate (DMP) during production of Bacillus thuringiensis based biopesticides," Journal of Hazardous Materials, vol. 171, no. 1-3, pp. 1016-1023, 2009.

[12] L. Ren, Y. Jia, N. Ruth et al., "Biodegradation of phthalic acid esters by a newly isolated Mycobacterium sp. YC-RL4 and the bioprocess with environmental samples," Environmental Science and Pollution Research, vol. 23, no. 16, pp. 16609-16619, 2016.

[13] B. Gao, P. Wang, H. Zhou et al., "Sorption of phthalic acid esters in two kinds of landfill leachates by the carbonaceous sorbents," Bioresource Technology, vol. 136, pp. 295-301, 2013.

[14] X. Zhou, J. Wei, H. Zhang, K. Liu, and H. Wang, "Adsorption of phthalic acid esters (PAEs) by amphiphilic polypropylene nonwoven from aqueous solution: the study of hydrophilic and hydrophobic microdomain," Journal of Hazardous Materials, vol. 273, pp. 61-69, 2014.

[15] P.-C. Huang, C.-J. Tien, Y.-M. Sun, C.-Y. Hsieh, and C.-C. Lee, "Occurrence of phthalates in sediment and biota: relationship to aquatic factors and the biota-sediment accumulation factor," Chemosphere, vol. 73, no. 4, pp. 539-544, 2008.

[16] S. Na, C. Jinhua, M. Cui, and J. Khim, "Sonophotolytic diethyl phthalate (DEP) degradation with UVC or VUV irradiation," Ultrasonics Sonochemistry, vol. 19, no. 5, pp. 1094-1098, 2012.

[17] J. D. Méndez-Díaz, M. M. Abdel daiem, J. Rivera-Utrilla, M. Sánchez-Polo, and I. Bautista-Toledo, "Adsorption/bioadsorption of phthalic acid, an organic micropollutant present in landfill leachates, on activated carbons," Journal of Colloid \& Interface Science, vol. 369, no. 1, pp. 358-365, 2012.

[18] Y. Jing, L. Li, Q. Zhang, P. Lu, P. Liu, and X. Lü, "Photocatalytic ozonation of dimethyl phthalate with $\mathrm{TiO} 2$ prepared by a hydrothermal method," Journal of Hazardous Materials, vol. 189, no. 1-2, pp. 40-47, 2011.

[19] G. C. C. Yang, S.-C. Huang, C.-L. Wang, and Y.-S. Jen, "Degradation of phthalate esters and acetaminophen in river sediments using the electrokinetic process integrated with a novel Fenton-like process catalyzed by nanoscale schwertmannite," Chemosphere, vol. 159, pp. 282-292, 2016.

[20] C. A. Staples, D. R. Peterson, T. F. Parkerton, and W. J. Adams, "The environmental fate of phthalate esters: a literature review," Chemosphere, vol. 35, no. 4, pp. 667-749, 1997.

[21] J. Boonnorat, C. Chiemchaisri, W. Chiemchaisri, and K. Yamamoto, "Removals of phenolic compounds and phthalic acid esters in landfill leachate by microbial sludge of two-stage membrane bioreactor," Journal of Hazardous Materials, vol. 277, pp. 93-101, 2014.

[22] N. S. Lewis and D. G. Nocera, "Powering the planet: chemical challenges in solar energy utilization," Proceedings of the National Academy of Sciences of the United States of America, vol. 103, no. 43, pp. 15729-15735, 2006.

[23] A. Melis, "Solar energy conversion efficiencies in photosynthesis: minimizing the chlorophyll antennae to maximize efficiency," Plant Science, vol. 177, no. 4, pp. 272-280, 2009.

[24] B. V. Chang, C. M. Yang, C. H. Cheng, and S. Y. Yuan, "Biodegradation of phthalate esters by two bacteria strains," Chemosphere, vol. 55, no. 4, pp. 533-538, 2004.

[25] S. R. Subashchandrabose, B. Ramakrishnan, M. Megharaj, K. Venkateswarlu, and R. Naidu, "Mixotrophic cyanobacteria and microalgae as distinctive biological agents for organic pollutant degradation," Environment International, vol. 51, pp. 59-72, 2013.
[26] J. Labarre, F. Chauvat, and P. Thuriaux, "Insertional mutagenesis by random cloning of antibiotic resistance genes into the genome of the cyanobacterium Synechocystis strain PCC 6803," Journal of Bacteriology, vol. 171, no. 6, pp. 3449-3457, 1989.

[27] N. F. Tsinoremas, A. K. Kutach, C. A. Strayer, and S. S. Golden, "Efficient gene transfer in Synechococcus sp. strains PCC 7942 and PCC 6301 by interspecies conjugation and chromosomal recombination," Journal of Bacteriology, vol. 176, no. 21, pp. 6764-6768, 1994.

[28] X. Zhang, D. M. Sherman, and L. A. Shermana, "The uptake hydrogenase in the unicellular diazotrophic cyanobacterium Cyanothece sp. strain PCC 7822 protects nitrogenase from oxygen toxicity," Journal of Bacteriology, vol. 196, no. 4, pp. 840849, 2014.

[29] J. Gao and J. Chi, "Biodegradation of phthalate acid esters by different marine microalgal species," Marine Pollution Bulletin, vol. 99, no. 1-2, pp. 70-75, 2015.

[30] B. Babu and J.-T. Wu, "Biodegradation of phthalate esters by cyanobacteria," Journal of Phycology, vol. 46, no. 6, pp. 1106-1113, 2010.

[31] A. Kapanen, J. R. Stephen, J. Brüggemann, A. Kiviranta, D. C. White, and M. Itävaara, "Diethyl phthalate in compost: ecotoxicological effects and response of the microbial community," Chemosphere, vol. 67, no. 11, pp. 2201-2209, 2007.

[32] C. A. Staples, W. J. Adams, T. F. Parkerton, and J. W. Gorsuch, "Aquatic toxicity of eighteen phthalate esters," Environmental Toxicology - Chemistry, vol. 16, no. 5, pp. 875-891, 1997.

[33] Y. Hai and L. Yun-Xia, "Dimethyl phthalate biodegradation by Dunaliella tertiolecta," Journal of Environmental Sciences, vol. 10, no. 3, pp. 41-46, 1998.

[34] L. Wang, C.-X. Wei, X. Yang, and L.-Y. Tang, "Di-(2-ethylhexyl) Phthalate toxic effect on Scenedesmus obliquus evaluated by the biomakers of inhibition rate and pigment content," in Proceedings of the 4th International Conference on Bioinformatics and Biomedical Engineering (iCBBE '10), Chengdu, China, June 2010.

[35] H. Yan, C. Ye, and C. Yin, "Kinetics of phthalate ester biodegradation by Chlorella pyrenoidosa," Environmental Toxicology and Chemistry, vol. 14, no. 6, pp. 931-938, 1995.

[36] J. Chi, H. Liu, B. Li, and G.-L. Huang, "Accumulation and biodegradation of dibutyl phthalate in Chlorella vulgaris," Bulletin of Environmental Contamination and Toxicology, vol. 77, no. 1, pp. 21-29, 2006.

[37] F. Zeng, K. Cui, X. Li, J. Fu, and G. Sheng, "Biodegradation kinetics of phthalate esters by Pseudomonas fluoresences FS1," Process Biochemistry, vol. 39, no. 9, pp. 1125-1129, 2004.

[38] J. Li, J.-A. Chen, Q. Zhao, X. Li, and W. Shu, "Bioremediation of environmental endocrine disruptor di-n-butyl phthalate ester by Rhodococcus ruber," Chemosphere, vol. 65, no. 9, pp. 16271633, 2006.

[39] X. Wu, Y. Wang, R. Liang, Q. Dai, D. Jin, and W. Chao, "Biodegradation of an endocrine-disrupting chemical di-nbutyl phthalate by newly isolated Agrobacterium sp. and the biochemical pathway," Process Biochemistry, vol. 46, no. 5, pp. 1090-1094, 2011.

[40] J. H. Niazi, D. T. Prasad, and T. B. Karegoudar, "Initial degradation of dimethylphthalate by esterases from Bacillusspecies," FEMS Microbiology Letters, vol. 196, no. 2, pp. 201-205, 2001.

[41] T. Kaneko, S. Sato, H. Kotani et al., "Sequence analysis of the genome of the unicellular cyanobacterium Synechocystis sp. strain PCC6803. II. Sequence determination of the entire genome and assignment of potential protein-coding regions," DNA Research, vol. 3, no. 3, pp. 109-136, 1996. 

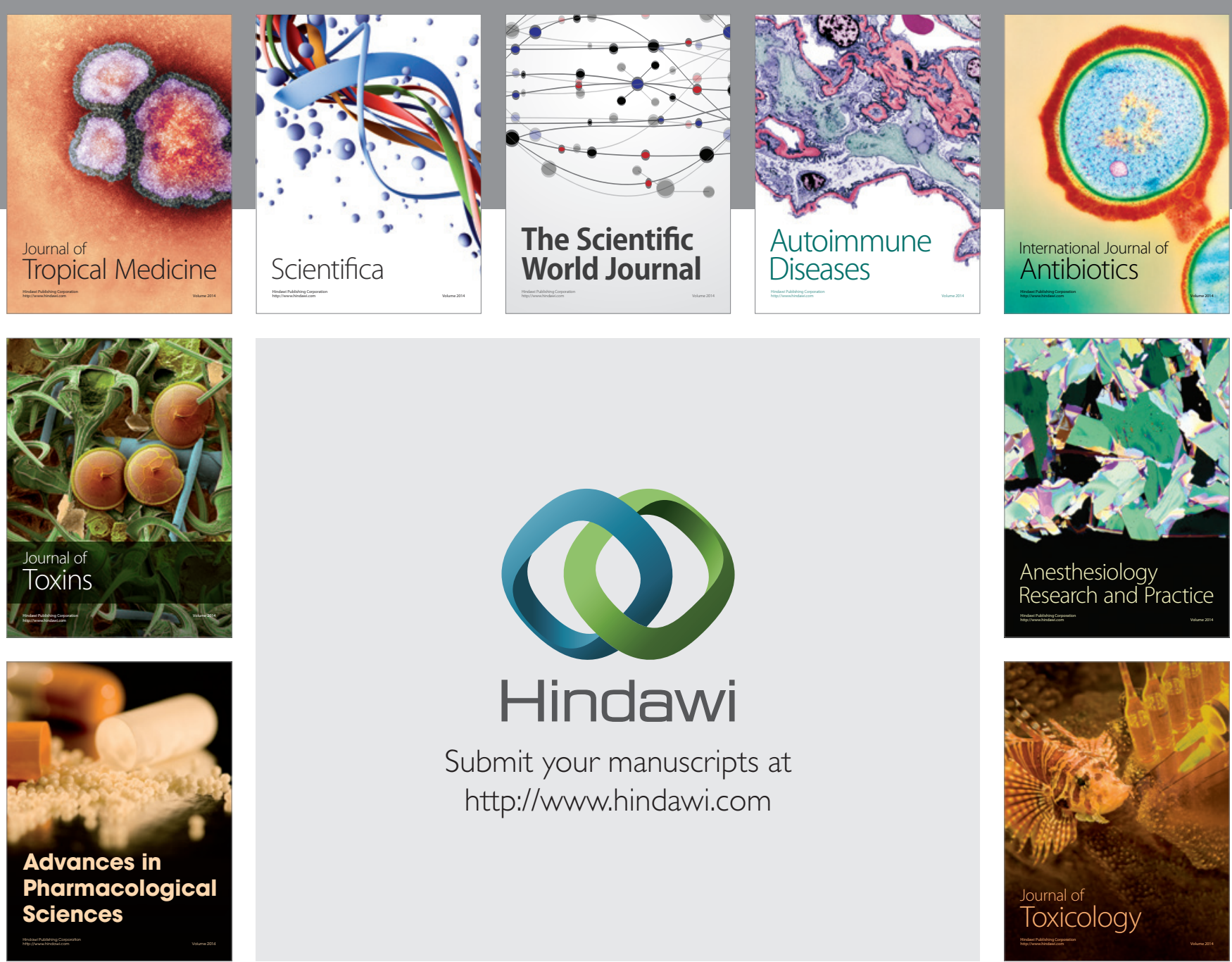

\section{Hindawi}

Submit your manuscripts at

http://www.hindawi.com
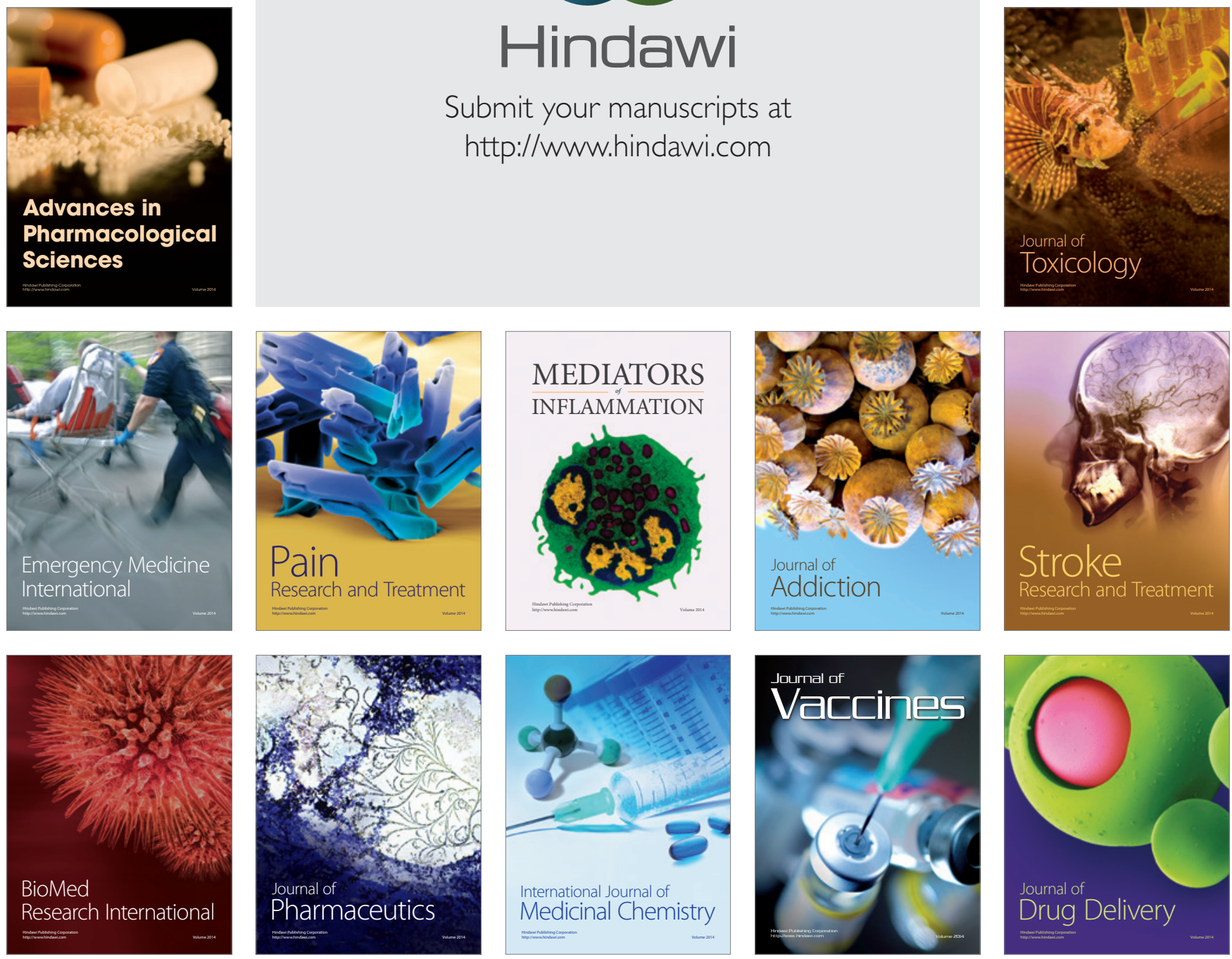